\title{
Aplicação da Escala de Equilíbrio de Berg para verificação do equilíbrio de idosos em diferentes fases do envelhecimento
}

\author{
Beatriz Bastos Dias"; Roberta da Silva Mota"; Taís Capobianco Gênova"; Vanessa Tamborelli"; \\ Vanessa Vieira Pereira"; Paulo de Tarso Puccini" ${ }^{* *}$
}

\section{Resumo}

Atualmente, sabe-se que um dos principais fatores que limitam a vida do idoso é o desequilíbrio. Este estudo tem o objetivo de observar, pela aplicação da Escala de Equilíbrio de Berg, qual a faixa etária, o agrupamento e a tarefa do teste em que o idoso apresenta maior déficit de equilíbrio. A amostra foi composta por 55 idosos, divididos em dois grupos: o primeiro composto por 30 idosos com faixa etária entre 65 e 79 anos e o segundo, com 25 idosos com faixa etária entre 80 e 94 anos. Para a análise dos dados a Berg foi dividida em cinco agrupamentos: transferências, provas estacionárias, alcance funcional, componentes rotacionais e base de sustentação diminuída. Nesta amostra, a faixa etária acima de oitenta anos foi a que apresentou maior desequilíbrio, com pontuação total da Berg indicativa de limite para risco de quedas. Em relação aos agrupamentos e tarefas da
Berg que mostraram maior desequilíbrio foram, respectivamente, a base de sustentação diminuída e a tarefa 14. Acredita-se que a prevenção seja importante nesta faixa etária e sugerem-se mais estudos para verificar se a reabilitação fisioterapêutica e exercícios físicos visando ao equilíbrio melhoram a pontuação total da Berg nesta faixa etária suficientemente para sair do limite de risco de quedas.

Palavras-chave: Idoso. Equilíbrio postural. Avaliação geriátrica. Maleabilidade.

* Fisioterapeuta. Especialista em atendimento interdisciplinar em Geriatria e Gerontologia do Hospital do Servidor Público Estadual - Francisco Morato de Oliveira. Endereço para correspondência: Beatriz Bastos Dias, Hospital do Servidor Público Estadual Francisco Morato de Oliveira, Ambulatório de Geriatria e Crônicos, Rua Pedro de Toledo, 1800, Vila Clementino, CEP 04039-901 São Paulo - SP. E-mail: bia_triz2004@ yahoo.com.br.

** Fisioterapeuta. Especialista em atendimento interdisciplinar em Geriatria e Gerontologia do Hospital do Servidor Público Estadual Francisco Morato de Oliveira.

*** Fisioterapeuta. Mestranda em Ciências da Saúde pelo Hospital do Servidor Público Estadual Francisco Morato de Oliveira. Especialista e supervisora do curso de aprimoramento em Geriatria e Gerontologia do Hospital do Servidor Público Estadual Francisco Morato de Oliveira.

**** Médico. Doutor em Saúde Pública pela Universidade de São Paulo.

$\hookrightarrow$ Recebido em julho de 2008 - Avaliado em abril de 2009.

$\longrightarrow$ doi: $10.5335 /$ rbceh.2009.020 


\section{Introdução}

O envelhecimento manifesta-se por declínio das funções dos diversos órgãos e sistemas, que caracteristicamente tendem a ser lineares em função do tempo, não se conseguindo definir um ponto exato de transição. O termo "idoso" refere-se à pessoa com 65 anos em países desenvolvidos e com sessenta anos nos países em desenvolvimento. (PAPALÉO NETTO; PONTE, 1996; PAPALÉO NETTO, 2006).

Nem todas as pessoas chegam à velhice nas mesmas condições: umas são mais vigorosas, mais autônomas e mais desenvolvidas do que outras, que não conseguem conservar seu dinamismo. Assim, alguns idosos estão mais propensos do que outros a diversas condições patológicas. Um idoso fragilizado por qualquer enfermidade, especialmente as que levam a alterações da mobilidade, equilíbrio e controle postural, ou mesmo a uma perturbação do equilíbrio, fica predisposto a quedas. (SOARES et al., 2003; RUWER; ROSSI; SIMON, 2005).

A queda é considerada um evento crítico na vida do idoso, sendo o acidente mais frequente entre eles, que pode gerar desde escoriações leves até limitações de atividade de vida diária (AVDs), fraturas, medo de quedas, perda de independência funcional, imobilidade, isolamento social. Em razão das suas complicações, é considerada a principal causa de morte naqueles com idade superior a 65 anos. Seu risco aumenta com o passar dos anos e sua maior prevalência é no sexo feminino, explicada pelo fato de as mulheres apresentarem média de ida- de mais elevada que os homens, podendo, assim, ter mais chances de distúrbios de equilíbrio. (RUWER; ROSSI; SIMON, 2005; VANDEVOORT, 2000; MACIEL; GUERRA, 2005; BARAÚNA et al., 2004; FRANCIULLI et al., 2007; SOUTHIARD et al., 2005).

Nos idosos, as quedas estão intimamente relacionadas com a marcha e a postura. Com o envelhecimento, podem ocorrer distúrbios no controle motor e na marcha, acarretando maiores chances de perturbações autoinduzidas. O risco de quedas aumenta no idoso em comparação a um indivíduo jovem, pois sabe-se que, com o avançar da idade, há uma diminuição na quantidade e qualidade das informações enviadas ao sistema nervoso central; a falta de um controle postural eficiente reduz seu equilíbrio, tornando-o mais suscetível a quedas, mesmo em situações mínimas de desestabilização. Outro fator que pode contribuir para o risco de quedas é a diminuição da força muscular. (PAIXÃO JR.; HECKMAN, 2002; GAZZOLA et al., 2006; BALLARD et al., 2004)

Para Arfken et al. (1994), dentre os idosos que relataram grande medo de quedas, $91 \%$ reportaram ao menos uma característica de fragilidade, $22 \%$ relataram demora para se levantar após uma queda e $85 \%$ teriam equilíbrio prejudicado.

O equilíbrio, ou controle postural, pode ser definido como o "processo pelo qual o Sistema Nervoso Central gera os padrões de atividade muscular necessários para regular a relação entre o centro de gravidade e a base de suporte". (PAIXÃO JR.; HECKMAN, 2002, p. 950). 
Esse equilíbrio pode ser considerado de duas formas: a primeira, quando o indivíduo se encontra na postura ereta, denominado "equilíbrio estático", ou seja, quando consegue manter seu centro de gravidade sobre a sua base de suporte; a outra, durante a marcha, denominada de "equilíbrio dinâmico". Quando o corpo está em equilíbrio estável e é deslocado por uma força externa, pode reagir de três maneiras: retornar a sua posição original, ir para uma nova posição e mover-se para longe da posição original, denominados, respectivamente, de equilíbrio estável, neutro e instável. (PAIXÃO JR.; HECKMAN, 2002; HAMILL; KNUTZEN, 1999; SANVITO, 2000).

Os sistemas responsáveis pelo controle postural são sensorial (visão, somatossensorial e vestibular), efetor (força, amplitude de movimento e alinhamento biomecânico) e processamento central. Esses sistemas podem sofrer influências decorrentes das alterações fisiológicas do envelhecimento, doenças crônicas, interações farmacológicas ou disfunções específicas. (GAZZOLA et al., 2005, 2006; MOCHIZUKI; AMADIO, 2003).

A força da gravidade e outras forças desestabilizadoras devidas ao movimento do corpo e sua interação com o ambiente colaboram para a instabilidade postural do indivíduo. (PAIXÃO JR.; HECKMAN, 2002).

Hoje, sabe-se que um dos principais fatores que limitam a vida do idoso é o desequilíbrio. Estima-se que a prevalência de queixas de equilíbrio nos idosos acima de 65 anos chegue a $85 \%$, podendo se manifestar como desequilíbrio, desvio de marcha, instabilidade, náuseas e quedas frequentes. (RUWER;
ROSSI; SIMON, 2005; SIMOCELLI et al., 2003).

Atualmente, não há um consenso na literatura sobre qual a idade ou faixa etária em que os idosos começam a perder o equilíbrio. Porém, Bittar et al. (2002) citam em seu trabalho que em mais da metade dos casos o desequilíbrio é visto como consequência de um comprometimento do sistema de equilíbrio como um todo, tendo origem, aproximadamente, entre os 65 e os 75 anos, e cerca de $30 \%$ dos idosos apresentam os sintomas nesta idade.

Portanto, tendo em vista o envelhecimento como um acontecimento inevitável, acompanhado de declínio funcional, com consequentes alterações posturais de equilíbrio, episódios de queda, e o fato de mais idosos chegarem a idades mais avançadas, percebeu-se a importância do tratamento multiprofissional, interdisciplinar e também da prevenção. Assim, este estudo tem o objetivo de observar na amostra delimitada, pela aplicação de uma avaliação do equilíbrio - escala de equilíbrio de Berg -, a faixa etária, o agrupamento e a tarefa do teste em que o idoso apresenta maior déficit de equilíbrio. Assim, podem-se idenficar as tarefas com maior dificuldade de execução e, consequentemente, as atividades de vida diária que podem ser afetadas, sabendo qual é o melhor momento para atuar (com o objetivo de manter ou melhorar esse equilíbrio, dependendo da faixa etária) e quais são os melhores recursos para poder intervir nessas tarefas prejudicadas, a fim de evitar, posteriormente, uma série de complicações e melhorar sua qualidade de vida. 


\section{Metodologia}

O estudo foi realizado no ambulatório do serviço de Geriatria e Gerontologia do Hospital do Servidor Público Estadual Francisco Morato de Oliveira (HSPE), localizado no município de São Paulo. O tipo de pesquisa utilizada foi a quantitativa, transversal, observacional, descritiva. A amostra foi composta por 55 indivíduos idosos (44 do sexo feminino e 11 do masculino), que foram divididos em dois grupos: um composto de trinta idosos na faixa etária de 65-79 anos (26 do sexo feminino e 4 do sexo masculino) e o outro com 25 idosos na faixa etária de 80-94 anos (18 do sexo feminino e 7 do masculino).

Como a idade mínima para atendimento no ambulatório de geriatria é a partir dos 65 anos, neste estudo optouse pela classificação de idoso como a pessoa com 65 anos ou mais. A média de idade nesta amostra foi de 79,0 e o desvio-padrão, de 6,6. Para participar da pesquisa todos tinham de ter deambulação independente, estar em acompanhamento no HSPE e assinar o termo de livre consentimento esclarecido. O trabalho foi avaliado pelo Comitê de Ética do HSPE, sendo aprovado pelo protocolo no 6907 .

Os critérios de exclusão foram determinados por meio de análise da hipótese diagnóstica colhida em prontuários de todos os voluntários. Assim, foram excluídos indivíduos idosos com patologias que interferissem em seu equilíbrio ou déficit cognitivo que prejudicasse a realização do teste, como vestibulopatias, demências, Parkinson e AVE, assim como limitações físicas e sensoriais, grande déficit visual ou auditivo, além de idosos com locomoção exclusivamente por cadeira de rodas.

Neste trabalho optou-se pela Escala de Berg, não por outros métodos de avaliação, porque, além de ser de baixo custo, de fácil administração e segura para pacientes idosos, é bastante utilizada na prática clínica e em pesquisa. Também atende a várias propostas, como acompanhamento do progresso dos pacientes, avaliação da efetividade das intervenções na prática clínica e em pesquisa e descrição quantitativa da habilidade de equilíbrio funcional. Berg e Nirman (1996) citam os estudos de Whitney et al., os quais, realizando pesquisa de revisão bibliográfica, evidenciaram que a escala de Berg, comparada a outros instrumentos, apresenta maior confiabilidade e validade quando utilizada em pesquisas científicas. (GAZZOLA et al., 2006; CONRADSSON et al., 2007).

A Escala de Berg é um instrumento validado, de avaliação funcional do equilíbrio composta de 14 tarefas com cinco itens cada e pontuação de 0-4 para cada tarefa: 0 - é incapaz de realizar a tarefa e 4 - realiza a tarefa independente. O escore total varia de $0-56$ pontos. Quanto menor for a pontuação, maior é o risco para quedas; quanto maior, melhor o desempenho. (GAZZOLA et al., 2006; CHRISTOFOLETT et al., 2006). A escala foi adaptada para aplicação no Brasil por Miyamoto et al. (2004), apresentando em cada item escores de 0-4 e um tempo determinado para cada tarefa; tem como pontuação para risco de quedas escore abaixo de 45 pontos. 
Neste estudo a aplicação da Escala de Berg foi realizada por uma fisioterapeuta. Os materiais utilizados foram cronômetro, fita métrica, cadeira com e sem braço e escada com $20 \mathrm{~cm}$ de altura.

Para a realização da análise dos dados, a Berg foi dividida em grupos com tarefas funcionais semelhantes: transferências (questões 1, 4 e 5), provas estacionárias (questões 2, 3, 6 e 7), alcance funcional (questão 8), componentes rotacionais (questões 9,10 e 11) e base de sustentação diminuída (questões 12 , 13 e 14). (FRANCIULLI et al., 2007; GAZZOLA et al. 2004).

O teste utilizado para análise estatística dos dados foi o teste $\mathrm{t}$ ou o teste $\mathrm{U}$, após a verificação da normalidade das variáveis pelo teste de KolmogorovSmirnov. O programa utilizado foi o SPSS 10.0, Windows. O estudo admitiu o valor de $\alpha<0,05$ para significância estatística.

\section{Resultados}

Comparando-se as faixas etárias (65 a 79 anos [Fx1] e 80 a 94 anos [Fx2]) e a média de pontuação total da Berg, obtiveram-se os seguintes resultados: a Fx1 apresentou média de perda de pontuação de 5 pontos, totalizando 51 pontos, e a Fx2, média de perda de pontuação de 10,9 pontos, totalizando 45,2 pontos. Também se compararam as faixas etárias e os grupos da Berg. Observando os dados, as variáveis faixa etária e média de pontuação da Berg mostraram-se estatisticamente significantes, com uma diferença de variação de 5,9 para um $\mathrm{p}<0,0001$ (Tab. 1).

Tabela 1 - Comparação da perda de pontuação na Escala Berg entre as faixas etárias, segundo grupos de tarefas da Escala Berg. São Paulo, 2007.

\begin{tabular}{l|c|c|c|c|c|c|c}
\hline \multirow{2}{*}{$\begin{array}{c}\text { Grupos de tarefa } \\
\text { Escala Berg }\end{array}$} & \multirow{2}{*}{$\begin{array}{c}\text { Pontuação } \\
\text { máxima }\end{array}$} & $\begin{array}{c}\text { Média de perda de } \\
\text { pontos na faixa etária }\end{array}$ & $\begin{array}{c}\text { Diferença de perda de } \\
\text { pontos entre as faixas }\end{array}$ & \multicolumn{2}{c}{$p$-valor } \\
\cline { 3 - 8 } & 65 a 79 & 80 a 94 & $X$ & $\%$ & Teste t & Teste U \\
\hline $\begin{array}{l}\text { Berg (todos os } \\
\text { grupos) }\end{array}$ & 56 & 5,0 & 10,9 & 5,9 & 11,0 & $<0,001$ & \\
Base de sustentação & 12 & 2,8 & 5,48 & 2,7 & 22,1 & 0,002 & \\
Componentes & 12 & 0,9 & 2,36 & 1,5 & 12,4 & $<0,001$ \\
rotacionais & 4 & 0,8 & 1,0 & 0,2 & 5,3 & & 0,081 \\
Alcance funcional & 16 & 0,1 & 0,6 & 0,5 & 3,1 & & 0,023 \\
Provas estacionárias & 12 & 0,4 & 1,4 & 1,0 & 8,7 & & 0,001 \\
\hline Transferências & & & & & &
\end{tabular}

Analisando os dados estatísticos conforme p-valor encontrado, dos agrupamentos das tarefas da Berg o único grupo que não apresentou estatística significante foi o alcance funcional. 
Tabela 2 - Diferença entre os agrupamentos e as faixas etárias. São Paulo, 2007.

\begin{tabular}{l|c|c|c|c|c}
\hline \multirow{2}{*}{$\begin{array}{c}\text { Agrupamento das } \\
\text { tarefas da Berg }\end{array}$} & \multicolumn{2}{|c|}{$\begin{array}{c}\text { Média por } \\
\text { faixa etária }\end{array}$} & \multirow{2}{*}{$\begin{array}{c}\text { Diferença } \\
\text { Fx2 e Fx1 }\end{array}$} & p-valor & $\begin{array}{c}\text { Variação \% } \\
\text { (Fx2 e Fx1) }\end{array}$ \\
\cline { 2 - 3 } & Fx1 & Fx2 & & & 8,67 \\
Transferências & 0,40 & 1,40 & 1,00 & 0,001 & 3,06 \\
Provas estacionárias & 0,06 & 0,56 & 0,50 & 0,044 & 5,25 \\
Alcance funcional & 0,83 & 1,04 & 0,20 & 0,273 & 12,41 \\
Componentes rotacionais & 0,86 & 2,36 & 1,49 & $<0,001$ & $22,08^{*}$ \\
Base de sustentação & 2,83 & 5,48 & 2,65 & 0,002 & \\
\hline
\end{tabular}

Nota: Grupo de tarefa com p-valor significativo e maior variação percentual entre as faixas etárias $(22,1 \%)$.

Observando os dados da Tabela 2, o grupo que obteve maior significância foi o agrupamento base de sustentação diminuída. Quanto à diferença da média em relação às faixas etárias por agrupamento, a faixa etária 2 (de 80-94 anos, ou Fx2) mostrou pontuação maior em todos os grupos em relação à faixa etária 1 (65-79, ou Fx1).
Em virtude de o grupo base de sustentação diminuída ter apresentado maior variação, verificou-se neste grupo qual das tarefas apresentara estatística significante maior. Para isso observaram-se as tarefas isoladamente, sendo o resultado encontrado maior significância na tarefa 14 com um $\mathrm{p}=0,003$. (Tab. 3).

Tabela 3 - Comparativo da média de pontuação entre as tarefas do grupo base de sustentação, segundo faixas etárias. São Paulo, 2007.

\begin{tabular}{l|c|c}
\hline \multicolumn{1}{c|}{ Tarefas } & $\begin{array}{c}\text { Diferença da } \\
\text { média }\end{array}$ & $\begin{array}{c}\text { p-valor da diferença } \\
\text { pelo Teste u }\end{array}$ \\
\hline Tocar banquinho & 0,62 & 0,015 \\
Em pé sem apoio com um pé à frente & 0,96 & 0,022 \\
Ficar sobre uma perna & 1,06 & 0,003 \\
\hline
\end{tabular}

\section{Discussão}

Neste estudo observou-se correlação estatisticamente significante entre faixa etária e Escala de Equilíbrio de Berg, mostrando uma mediana de pontuação da BBS significativamente menor no grupo com a faixa etária igual ou maior que oitenta anos, o que indica maior perda de equilíbrio nesta faixa etária. Esse achado corrobora com Gazzola et al. (2006), que encontraram uma mediana da pontuação da BBS significativamen- te menor no grupo com faixa etária de oitenta anos ou mais. Em seu estudo, observaram-se numa população de 120 idosos com disfunção vestibular crônica em atendimento ambulatorial a associação com o equilíbrio funcional, avaliado pela Berg Balance Scale (BBS), os dados sociodemográficos, clínicos, de mobilidade (Timed Up and Go-TUG) e de marcha (Dynamic Gait Index-DGI).

Maciel e Guerra (2005) relataram correlação com o desequilíbrio acima de 75 anos. Em seu estudo, observaram 
310 idosos da comunidade com idade acima de sessenta anos e analisaram as variáveis associadas com o distúrbio de equilíbrio. Essa correlação significativa entre a faixa etária e a Berg pode ser explicada em razão do próprio processo do envelhecimento, que compromete a habilidade do sistema nervoso central de realizar o processamento dos sistemas vestibular, proprioceptivo e visual. Com o aumento da idade, há alteração do controle postural em virtude da diminuição na velocidade de condução das informações, assim como no processamento das respostas, que são lentas e inadequadas, gerando instabilidade e pondo em risco a movimentação segura dos idosos, pela sua predisposição a quedas. (GAZZOLA et al. 2006; RUWER; ROSSI; SIMON, 2005; MACIEL; GUERRA, 2005; FARIA et al. 2003; PERRACINI, 1998; BARAÚNA et al. 2004).

A pontuação encontrada nessa faixa etária foi de 45,12, indicando, de acordo com a BBS, limite para risco de quedas. Em estudo realizado por Nakatani et al. (2003), investigaram-se o perfil sociodemográfico e a capacidade funcional dos idosos atendidos por uma equipe de saúde da família. A amostra foi composta por 107 indivíduos com idade igual ou superior a oitenta anos, com dificuldades de equilíbrio e mobilidade, as quais foram associadas a dependência em realizar as atividades de vida diária e atividades instrumentais de vida diária.

Vale et al. (2006) relatam em seu estudo que a perda da massa muscular ocorrida com o envelhecimento contribui para a diminuição da capacidade funcional, dificultando a execução das tarefas diárias.
Esses estudos podem mostrar que, com a função prejudicada, os idosos logo perderão a sua mobilidade, aumentando, assim, o risco para quedas. (BERG; NORMAN, 1996).

Em estudo realizado por Ozan et al. (2005) com 116 idosos acima de 65 anos, verificou-se a relação entre o risco de quedas (equilíbrio funcional, mobilidade, propriocepção, força muscular, flexibilidade e medo de quedas) e a qualidade de vida. Encontrou-se forte correlação positiva entre qualidade de vida e equilíbrio e força e correlação negativa entre qualidade de vida e medo de quedas e mobilidade funcional. Não houve correlação com os itens qualidade de vida e flexibilidade e propriocepção.

Verificou-se, portanto, que o maior desequilíbrio se encontra na faixa etária acima de oitenta anos, em que foi encontrada a pontuação 45 . Será que sessões de fisioterapia e exercícios físicos visando a uma melhora do equilíbrio aumentariam essa pontuação, fazendo-os sair do limite de risco de quedas? O estudo de Yokokawa et al. (2003) determinou os efeitos de um programa da prevenção de queda junto a pessoas idosas de 75 anos ou mais de uma comunidade rural no Japão. Os resultados mostraram que exercícios específicos poderiam melhorar alguns aspectos do desempenho físico. Como o programa de exercício foi eficaz para pessoas idosas de 75 anos ou mais, pode contribuir para diminuir as quedas e prolongar a vida independente.

Dentre os riscos para quedas estão o déficit de equilíbrio e a menor força em membros inferiores. (WONG et al., 2001). A fisioterapia, por meio de 
exercícios de fortalecimento, atua no sistema efetor, diminuindo seus déficits e podendo reduzir o risco para quedas. Este último pode ser diminuído principalmente pelo aumento da força nos membros inferiores, com o que os idosos podem manter seu controle postural em muitas situações. (FARIA et al., 2003).

Diversos estudos avaliando os efeitos dos programas de treinamento de força de membros inferiores a fim de verificar a melhora da força e do equilíbrio em idosos constataram resultados positivos, como aumento de força, equilíbrio, de desempenho de atividade de vida diária (AVD), mobilidade e flexibilidade. (BALLARD et al. 2004; VALE et al., 2006; HESS; WOOLLACOTT, 2005; GATTS; WOOLLACOTT, 2006). Para Faria et al. (2003), que realizaram uma revisão bibliográfica sobre o treinamento de força na reabilitação da função muscular, equilíbrio e mobilidade de idosos, mesmo em idades avançadas a implementação de um programa de exercício terapêutico minimiza ou evita o declínio funcional acentuado, prevenindo os efeitos das doenças ou amenizando-os.

Esse achado também corrobora com o estudo de revisão de Gazzola et al. (2005) sobre envelhecimento e sistema vestibular, porém os autores não especificaram a faixa etária, e com o estudo de Steadman, Donaldson e Karla (2003), os quais concluíram que o treinamento pode, além de melhorar a confiança desses pacientes, melhorar a sua qualidade de vida. Muitos trabalhos têm demonstrado a eficácia do treinamento de equilíbrio para o aumento do controle postural, que também é útil para prevenção e redução do número de quedas. (PERRACINI, 1998; WOLF et al., 2001; SHUMWAYCOOK; WOOLLACOTT, 2003). Dentre os exercícios físicos destaca-se o tai $c h i$, que, além de melhorar o equilíbrio, aumenta a força de membros inferiores. (WONG et al., 2001; GATTS; WOOLLACOTT, 2006; SHUMWAY-COOK; WOOLLACOTT, 2003).

Observando os agrupamentos das tarefas da Berg deste estudo, o único que não apresentou estatística significante foi o alcance funcional. Essa tarefa verifica a mobilidade e flexibilidade de tronco para frente e membros superiores; conforme sua pontuação, será preditivo para risco de quedas. Nesta amostra não houve diferença entre as faixas etárias, mostrando que ambas não tiveram alterações significativas. Uma das hipóteses para esse resultado é que com o envelhecimento, primeiramente, perde-se força nos membros inferiores e, posteriormente, nos membros superiores e tronco. Isso pode ser explicado em razão das tarefas realizadas diariamente e de a amostra constar de pessoas com deambulação independente, sem patologias que interfiram em seu equilíbrio. Sabe-se que com o avanço da idade ocorre perda da flexibilidade, a qual pode se relacionar com a dificuldade em utilizar escadas, levantar-se de cadeiras e, em alguns casos, deambular de modo independente. (LIMA et al., 2004). Verificando as dificuldades citadas, pode-se dizer que são tarefas nas quais se utilizam com maior ênfase os membros inferiores.

Analisando a Tabela 2, pode-se verificar que, entre as faixas etárias, a que apresentou maior média em todos os 
agrupamentos correspondeu aos idosos com idade igual ou superior a oitenta anos, indicando maior desequilíbrio nessa faixa etária.

Em relação aos agrupamentos, o que obteve maior diferença de média e diferença entre as faixas etárias 1 e 2 foi a base de sustentação diminuída, achado que pode ser visto na Tabela 2. Pode-se observar que os resultados encontrados para este grupo indicam que teve o maior prejuízo. Esse dado corrobora os achados de Gazzola et al. (2004), que em seu estudo objetivaram caracterizar $o$ comportamento funcional do equilíbrio e identificar o grau de associação do desempenho físico-funcional do equilíbrio a dados sociodemográficos, funcionais e clínicos. Foram avaliados 93 idosos acima de 65 anos e utilizou-se para verificar o equilíbrio funcional a escala de Berg (BBS), dividida e analisada em cinco dimensões: transferências, provas estacionárias, alcance funcional, componentes rotacionais e base de sustentação diminuída.

A base de sustentação diminuída é um agrupamento composto por três tarefas $(12,13,14)$ : 12 - transferência dinâmica de peso enquanto está em pé sem apoio - tocar um banquinho, o indivíduo deverá tocar oito vezes o banquinho alternadamente em até $20 \mathrm{~s} ; 13$ - permanecer em pé sem apoio com um pé à frente 30s; 14 - permanecer sobre uma perna por mais de 10 s. Neste trabalho optouse por analisar isoladamente somente as tarefas 12,13 e 14, em razão de seu agrupamento ter mostrado, em relação aos outros, maior variação. (MIYAMOTO et al., 2004; FRANCIULLI et al., 2007).
Analisando as tarefas, constatou-se que a mais prejudicada foi a 14 , corroborando com outros estudos. Para Taguchi e Santos (2007), que verificaram a tendência a quedas em 130 idosos ativos (60-79 anos) por meio de aplicação da Escala de Berg (BBS), a tarefa com maior comprometimento foi a 14, demonstrando que é eficiente para distinguir idosos ativos com tendência a quedas. Este achado também foi relatado no estudo de Wang et al. (2006), que verificaram em 268 idosos da comunidade acima de 65 anos as tarefas mais desafiadoras da Berg. De acordo com seus resultados, as tarefas 13 e 14 são as mais desafiadoras.

$\mathrm{O}$ artigo de Jonsson et al. (2004) menciona o equilíbrio unipodal como uma ferramenta clínica para avaliar o equilíbrio em pessoas com desordens no equilíbrio. A tarefa de ficar em pé sobre uma perna requer uma ação voluntária inicial de mover o centro de gravidade sobre a perna apoiada (fase dinâmica), seguido da tarefa de manter a orientação postural no espaço (fase estática). O teste do apoio unipodal é capaz de avaliar quantitativamente o equilíbrio estático. Contudo, como a habilidade de mudança de apoio bipodal para unipodal é necessária para inúmeras atividades de vida diária, como se virar, subir degraus, caminhar e vestir-se, a manutenção do equilíbrio estático é de pouca utilidade para se avaliar o equilíbrio em tais atividades. Nesse estudo foram testados tanto indivíduos idosos quanto jovens no teste de equilíbrio sobre uma perna. Os resultados sugerem que o grupo de idosos apresentou dificuldades em manter o apoio unipodal na fase estática por dificuldades em ajustar a posição unipodal 
na fase dinâmica. O estudo citou a pesquisa de Iverson et al., a qual mostrou que o tempo de permanência em apoio unipodal cresce conforme a força muscular aumenta. Assim, a dificuldade em se manter em um pé só pode depender dos componentes musculares, do ajuste postural ou de ambos.

\section{Conclusão}

Neste estudo verificou-se que a maior perda de equilíbrio encontra-se na faixa etária de oitenta anos ou mais. Nesta amostra essa faixa etária obteve pontuação da Berg indicativa de limite de risco para quedas. Entre os agrupamentos das tarefas da Berg o que apresentou maior comprometimento, sendo indicativo de maior desequilíbrio em idosos de oitenta anos ou mais, foi a base de sustentação diminuída e, entre as tarefas deste grupo, a 14 indicou nesta amostra ser a tarefa considerada de maior complexidade para sua execução entre os idosos de oitenta anos ou mais, sendo a de maior comprometimento.

Acredita-se que, como muitos idosos estão atingindo idades mais elevadas, a prevenção torna-se uma meta cada vez mais importante para essa faixa etária, pois com o aumento da idade e a perda de equilíbrio fisiológico o idoso passa a sofrer grande risco de quedas e suas complicações tornam-se cada vez mais significativas e difíceis de tratar.

Sugere-se que mais estudos sejam realizados a fim de verificar se a reabilitação fisioterapêutica ou exercícios físicos (como tai chi, hidroterapia, caminhada) visando ao equilíbrio em idosos acima de oitenta anos aumenta esse equilíbrio, melhorando o escore de pontuação total da Berg num nível suficiente para sair do limite de risco de quedas e, assim, a qualidade de vida. Sugerem-se trabalhos com um maior número de idosos a fim de dividi-los em mais faixas etárias, não somente duas, e estudo longitudinal.

Application of the Berg Balance Scale to check the balance of the elderly in different stages of aging

\section{Abstract}

Currently, one of the main factors that limit the life of the aged one it is the disequilibrium. This study has the objective to observe, through the application of the Scale of Balance of Berg, which the age, the grouping and the task of the test that the aged one presents greater balance deficit. The sample was composed for 55 aged ones; it was divided in two groups, the first group with 30 aged ones with 65-79 years and the second group with 25 aged ones with ages between 80-94 years. For the analysis of the data's Berg was divided in five groupings (stationary transferences, tests, functional reach, rotational components and base of diminished sustentation). In this sample, the ages above of 80 years was the one that presented greater disequilibrium being its total punctuation of the indicative Berg of limit for risk of falls. In relation to the groupings and tasks of the Berg that they had shown greater disequilibrium respectively had been: the sustentation base of diminished and task 14. It gives credit that the prevention is important in this ages; one suggests more studies to verify if the rehabilitation and physical exercises aiming at the balance, improve the total punctuation Berg in this ages to leave the limit for risk of falls enough.

Key words: Aged. Postural balance. Geriatric assessment. Pliability. 


\section{Referências}

ARFKEN, C. L. et al. The prevalence and correlates of fear of falling in elderly persons living in the community. Am. J. Public. Health, p. 565-570, 1994.

BALLARD, J. E. et al. The effect of 15 weeks of exercise on balance, leg strength, and reduction in falls in 40 women aged 65 to 89 years. J. Am. Med. Womens Assoc., v. 59, n. 4, p. 255-261, 2004.

BARAÚNA, M. A. et al. Estudo do equilíbrio estático de idosos e sua correlação com quedas. Fisioterapia Brasileira, v. 5, n. 2, p. 136-140, mar./abr. 2004.

BERG, K. O.; NORMAN, K. E. Functional assessment of balance and gait. Clinics in Geriatrics Medicine, v. 12, n. 4, p. 705-723, 1996.

BITTAR, R. S. M. et al. Síndrome do desequilíbrio no idoso. Pró-fono - Revista Atual Científica, v. 14, n. 1, p. 119-128, 2002.

CONRADSSON, M. et al. Berg balance scale: intrarater test-retest reliability among older people dependent in activities of daily living and living in residential care facilities. Phys. Ther., v. 87, n. 9, p. 1155-1563, Set. 2007.

CHRISTOFOLETT, G. Risco de quedas em idosos com doença de Parkinson e demência de Alzheimer: um estudo transversal. Revista Brasileira de Fisioterapia, [on-line], São Carlos, v. 10, n. 4, p. 429-433, out./dez. 2006.

FARIA, J. C. et al. Importância do treinamento de força na reabilitação da função muscular, equilíbrio e mobilidade de idosos. Acta Fsiatrica, v. 10, n. 3, p. 133-137, jan. 2003.

FRANCIULLI, S. E et al. A modalidade de assistência Centro-Dia Geriátrico: efeitos funcionais em seis meses de acompanhamento multiprofissional. Ciência Saúde Coletiva, Rio de Janeiro, v. 12, n. 2, p. 373-380, 2007. GATTS, S. K.; WOOLLACOTT, M. H. Neural mechanisms underlying balance improvement with short term Tai Chi training. Aging Clin. Exp. Res., v. 18, n. 1, p. 7-19, fev. 2006.
GAZZOLA, J. M. et al. Caracterização funcional do equilíbrio de idosos em serviço de reabilitação gerontológica. Revista de Fisioterapia - Universidade de São Paulo, São Paulo, v. 11, n. 1, p. 1-14, jan./jun. 2004.

GAZZOLA, J. M. et al. O envelhecimento e o sistema vestibular. Fisioterapia em Movimento, Paraná, v. 18, n. 3, p. 39-48, jul./ set. 2005.

GAZZOLA, J. M. et al. Fatores associados ao equilíbrio funcional em idosos com disfunção vestibular crônica. Revista Brasileira de Otorrinolaringologia, São Paulo, v. 72, n. 5, p. 683-690, set./out. 2006.

HAMILL, J.; KNUTZEN, K. M. Bases biomecânicas do movimento humano. São Paulo: Manole, 1999.

HESS, J. A.; WOOLLACOTT, M. Effect of high-intensity strength-training on functional measures of balance ability in balanceimpaired older adults. J. Manipulative Physiol. Ther., v. 28, n. 8, p. 582-590, Oct. 2005. JONSSON, E. et al. One leg stance in healthy young and elderly adults: a measure of postural steadiness. Clin. Biomech., v. 19, p. 688-694, Apr. 2004.

LIMA, L. A. O. et al. Estudo da confiabilidade de um instrumento de medida de flexibilidade de adultos e idosos. Revista de Fisioterapia - Universidade de São Paulo, São Paulo, v. 11, n. 2, p. 83-89, jul./dez. 2004.

MACIEL, A. C. C.; GUERRA R. O. Prevalência e fatores associados ao déficit de equilíbrio em idosos. Revista Brasileira de Ciência do Movimento, Brasília, v. 13, n. 1, p. 37-44, jan. 2005.

MIYAMOTO, S. T. Brazilian version of the Berg balance scale. Braz. Med. Biol. Res., Ribeirão Preto, v. 37, n. 9, p. 1411-1421, abr. 2004.

MOCHIZUKI, L.; AMADIO, A. C. As funções do controle postural durante a postura ereta. Revista de Fisioterapia - Universidade de São Paulo, São Paulo, v. 10, n. 1, p. 7-15, jan./jun. 2003.

NAKATANI, A. Y. K. et al. Perfil sóciodemográfico e avaliação funcional de idosos 
atendidos por uma equipe de saúde da Família na periferia de Goiânia, Goiás. Revista da Sociedade Brasileira de Clínica Médica, São Paulo, v. 1, n. 5, p. 131-136, 2003.

OZAN, A. et al. The relationship between risk factors for falling and the quality of life in older adults. BMC Public. Health, v. 5, n. 90, p. 5-90, 2005.

PAIXÃO JR., C. M.; HECKMANN, M. Distúrbios da postura, marcha e quedas. In: FREITAS, E. V. et al. Tratado de geriatria e gerontologia. Rio de Janeiro: Guanabara Koogan, 2002. p. 950-960.

PAPALÉO NETTO, M.; PONTE, J. R. Envelhecimento: desafio na transição do século. In: PAPALÉO NETTO, M. Gerontologia. São Paulo: Atheneu, 1996. p. 3-12.

PAPALÉO NETTO, M. O estudo da velhice no século XX: histórico, definição do campo e termos básicos In: FREITAS, E.V. et al. (Org.). Tratado de geriatria e gerontologia. 2. ed. Rio de Janeiro: Guanabara Koogan, 2006. p. 2-12.

PERRACINI, M. R. Equilíbrio e controle postural em idosos. Revista Brasileira de Postura e Movimento, São Paulo, v. 2, n. 4, p. 130-142, 1998.

RUWER, S. L.; ROSSI, A. G.; SIMON, L. F. Equilíbrio no idoso. Revista Brasileira de Otorrinolaringologia, São Paulo, v. 71, n. 3, p. 298-303, maio/jun. 2005.

SANVITO, W. L. Propedêutica neurológica básica. São Paulo: Atheneu, 2000.

SHUMWAY-COOK, A.; WOOLLACOTT, M. B. Envelhecimento e controle postural In: Controle motor: teorias e aplicações práticas. 2. ed. São Paulo: Manole, 2000. p. 228-230.

SIMOCELLI, L. et al. Perfil diagnóstico do idoso portador de desequilíbrio corporal: resultados preliminares. Revista Brasileira de Otorrinolaringologia, São Paulo, v. 69, n. 6, p. 772-777, nov./dez. 2003.

STEADMAN, J.; DONALDSON, N.; KARLA, L. A. randomized controlled trial of an enhanced balance training program to improve mobility and reduce falls in elderly patients. J. Am. Geriatr. Soc., v. 51, n. 6, p. 847-852, Jun. 2003.

SOARES, A. V. et al. Estudo comparativo sobre propensão de quedas em idosos institucionalizados e não institucionalizados através do nível de mobilidade funcional. Fisioterapia Brasil, São Paulo, v. 4, n. 1, p. 13-17, 2003.

SOUTHIARD, V. et al. The multiple tasks test as a predictor of falls in older adults. Gait Posture, v. 22, n. 4, p. 351-355, Dec. 2005.

TAGUCHI, C. K; SANTOS, R. D. Análise dos resultados da tendência a quedas (BBS) em idosos ativos, por meio da aplicação da Berg Balance Scale. Med. Reabil., v. 26, n. 2, p. 10-13, 2007.

VALE, S. G. S. et al. Efeitos do treinamento resistido na força máxima, na flexibilidade e na autonomia funcional de mulheres idosas. Revista Brasileira de Cineantropometria e Desempenho Humano, Florianópolis, v. 8, n. 4, p. 52-58, 2006.

VANDEVOORT, A. A. Alterações biológicas e fisiológicas. In: PICKLES, B. et al. Fisioterapia na terceira_idade. 2. ed. São Paulo: Santos, 2002. p. 85-130.

WANG, C. Y. et al. Psychometric properties of the Berg Balance Scale in a communitydwelling elderly resident population in Taiwan. J. Formos. Med. Assoc., v. 105, n. 12 , p. 992-1000, 2006.

WOLF, B. et al. Effects of a physical therapeutic intervention for balance problems in the elderly: a single-blind, randomized, controlled multicentre trial. Clin. Rehabil., v. 15, n. 6 , p. 624-636, Dec. 2001.

WONG, A. M. et al. Coordination exercise and postural stability in elderly people: effect os tai chi chuan. Arch. Phys. Med. Reabil., v. 82, p. 608-612, May 2001.

YOKOKAWA, Y. et al. Intervention study using a fall prevention program to prevent functional decline of old - old elderly in a rural community. Nippon Ronen Igakkai Zasshi, v. 40, n. 1, p. 47-52, Jan. 2003. 